\title{
PREMIUM RATES UNDER INFLATIONARY CONDITIONS
}

\author{
H. R. WATERS * \\ Heriot-Watt University, Edinburgh
}

\section{INTRODUCTION}

This short note has as its starting point an interesting article by TAYLOR (1979) in which he considered the effects of inflation on a risk process. Taylor showed that if the premium density increased at the same rate as the cost of individual claims then, under certain conditions, ultimate ruin was certain. This raises a natural question, viz. "If the cost of individual claims is increasing how should the premiums be increased in order to keep the probability of ruin under control ?"' It is this question that we shall be considering in this note.

In the next section we define the risk process that we shall be studying for the remainder of this note. Our process is essentially a compound Poisson process except that we allow the distribution function of an individual claim to depend on the time at which the claim occurs. We start the third section by deriving, with the help of a general result of GERBER (1973), a formula for the future premium density that will keep the probability of ruin for our process below a predetermined bound. We then derive a simple approximation to this formula that shows more clearly how we require the premium density to change in relation to the change in claims costs. Finally we show that if we consider the same process with annual premiums then the probability of ultimate ruin will be kept below a predetermined bound if the annual premium is calculated according to the principle of zero utility with an exponential utility function or, as a first approximation, according to the variance principle.

\section{THE RISK PROCESS}

In this section we describe the risk process that we shall be studying in this note.

We assume claims are independent of each other and occur as the points of a Poisson process with a mean rate of $p$ claims each year. The amount of a single claim occurring at time $t$ years has distribution function $F_{t}$ where

$$
F_{t}(x)=F_{0}(x / i(t)) \quad t \geqslant 0
$$

and where $i(t)(>0)$ is a non-stochastic index of claims inflation at time $t$ and

* The author is grateful to the referee for some useful comments on an earlier draft of this paper. 
$i(0)=1$. If we denote the moment generating function of $F_{t}$ by $m_{t}$ it is clear that

$$
m_{t}(\theta)=m_{0}(\theta i(t))
$$

We shall assume that $m_{0}(\theta)$ is finite for all values of $\theta$ but note the remarks following the proof of the theorem in the next section.

We denote by $X_{t}$ the accumulated claims in the interval $[0, t]$. The moment generating function of $X_{t}$ can be shown to be

$$
M_{t}(\theta)=\exp \left\{p t\left[1 / t \int_{0}^{1} m_{s}(\theta) d s-1\right]\right\}
$$

For a derivation of this formula see either BüHLMANN (1970, p. 60) or TAYLOR (1979, p. 153). For $n=1,2,3 \ldots$ we define

$$
Y_{n}=X_{n}-X_{n-1}
$$

so that $Y_{n}$ is the total claims in the $n$-th year. Using (3) it is easy to show that

$$
\begin{gathered}
E\left[Y_{n}\right]=p m \int_{n-1}^{n} i(s) d s \\
\operatorname{Var}\left[Y_{n}\right]=p \alpha \int_{n-1}^{n} i^{2}(s) d s
\end{gathered}
$$

where $m$ and $\alpha$ are the first and second moments of $F_{0}$ about the origin respectively.

We denote by $U$ the insurer's free reserves at $t=0$. We make no specific allowance for investment income to be added to the insurer's reserves but following the remarks on p. 161 of TAYLOR (1979) we can regard $i(t)$ as $i_{1}(t) / i_{2}(t)$ where $i_{1}(t)$ is a true index of claims inflation at time $t$ and $i_{2}(t)$ is the accumulated amount at time $t$ of a unit sum invested at time $o$.

TAYLOR (1979) has shown that if the total premium income in $[0, t]$ is

$$
C_{t}=c \int_{0}^{t} i(s) d s
$$

for some constant $c$ then ultimate ruin is certain for our risk process provided only that there exists some constant $k$ such that

$$
F_{0}(c / k)<1 \text { and } \int_{0}^{t} i(s) d s \leqslant k i(t) \text { for all } t \geqslant 0 .
$$

i.e. provided only that sufficiently large claims are possible and that the rate of inflation is large enough. The above conditions on $F_{\mathbf{0}}$ and $i(s)$ are not necessary for the results of the next section. 


\section{PREMIUM RATES}

We denote by $c_{t}$ the insurer's instantaneous rate of premium income at time $t$. We start this section by showing how to determine $c_{t}$ in such a way that the insurer's probability of ultimate ruin can be kept below a predetermined bound. We do this in the following theorem.

\section{Theorem}

The insurer's probability of ultimate ruin will be bounded above by $\exp \{-R U\}$ if $c_{t}$ is chosen so that

$$
c_{t}=p\left[m_{t}(R)-1\right] / R \quad \text { for } t \geqslant 0
$$

where $R$ is any positive number.

Proof

We define the process $\left\{Z_{t}\right\}_{t} \geqslant 0$ by

$$
Z_{t}=\int_{0}^{1} c_{s} d s-X_{t}
$$

(where $c_{s}$ is as defined in (9)) so that the insurer's reserves at time $t$ are $U+Z_{t}$. This process has independent increments so we can use a result of GERBER (1973) which states that the probability of ultimate ruin for such a process is bounded above by

$$
\min _{r} \exp \{-r U\} \max _{0 \leqslant t} E\left[\exp \left\{-r Z_{t}\right\}\right]
$$

But, using (3), we have for any $r$

$$
\begin{aligned}
& E\left[\exp \left\{-r Z_{t}\right\}\right]=\exp \left\{-r \int_{0}^{t} c_{s} d s\right\} M_{t}(r) \\
& =\exp \left\{-r \int_{0}^{t} c_{s} d s+p t\left[1 / t \int_{0}^{t} m_{s}(r) d s-1\right]\right\} \\
& =\exp \left\{-\int_{0}^{t}\left[p+r c_{s}-p m_{s}(r)\right] d s\right\}
\end{aligned}
$$

By putting $r=R$ in (12) and then using (9) we can see that

$$
E\left[\exp \left\{-R Z_{t}\right\}\right]=1 \quad \text { for all } t \geqslant 0 .
$$

Our theorem is then a simple consequence of Gerber's result.

\section{Remarks}

1. In the special case $i(s)=1$ for all $s$ (i.e. for a standard compound Poisson risk process) the above theorem is nothing more than Lundberg's inequality 
for the probability of ruin since it is clear from (9) that $R$ is the insurer's insolvency constant. What we have done is to extend this result to the case where the cost of a claim depends on the time at which it occurs and we have achieved this by requiring $c_{t}$ to be calculated in such a way that the insurer's "instantaneous insolvency constant" at time $t$ is held fixed at some value $R>0$.

2. An alternative interpretation of our result is that we have chosen $c_{t}$ in such a way that the process $\left\{\exp \left(-R Z_{t}\right)\right\}_{t \geqslant 0}$ is a martingale. See Gerber (1975).

3. The assumption that $m_{0}(\theta)$ is finite for all $\theta$ implies that $m_{t}(R)$ and hence $c_{t}$ will be finite for any values of $R$ and $t$. Suppose that only the weaker condition

$$
m_{t}(\theta)<\infty \quad \text { for all } 0 \leqslant \theta \leqslant \theta_{0} \text { and } 0 \leqslant t \leqslant t_{0}
$$

holds where $\theta_{0}, t_{0}>0$. We can then show that the probability of ruin before time $t_{0}$ is bounded above by $\exp \{-R U\}$ provided $R \leqslant \theta_{0}$ and provided $c_{t}$ is calculated as in $(9)$ for $0 \leqslant t \leqslant t_{0}$. The proof is as before except that it requires the finite-time version of Gerber's result. See p. 207 of GERBER (1973).

Formula (9) gives little indication of the way in which we require $c_{t}$ to change relative to $i(t)$. We try to provide this, at least for small values of $t$, in the following corollary.

\section{Corollary 1}

The rate of premium income $c_{t}$ specified by (9) gives the following approximation for small values of $t$ :

$$
c_{t} \doteqdot c_{0} i(t)[1+\lambda i(t)] /[1+\lambda]
$$

where $\lambda=R \alpha / 2 m$.

Proof

Formula (9) gives

$$
c_{t}=c_{0}\left[m_{t}(R)-1\right] /\left[m_{0}(R)-1\right]
$$

and we have

$$
m_{t}(R)-1=R i(t) m+\alpha(R i(t))^{2} / 2+\sum_{i=3}^{\infty}(R i(t))^{j} \alpha_{j} / j !
$$

where $\alpha_{j}$ is the $j$-th moment of $F_{0}$ about the origin. By assumption, (17) is a convergent series. In practice $R$ is likely to be small so that if $i(t)$ is not large the first two terms on the right hand side of (18) should give a reasonable approximation to $\left[m_{t}(R)-1\right]$. Making a similar approximation to $\left[m_{0}(R)-1\right]$ and putting these two approximations into (16) we obtain (15). 


\section{Remarks}

1. It is interesting to compare (15) with (7).

2. It can be easily checked that if $i(t) \geqslant 1$ and $m, \alpha, \alpha_{j} \geqslant 0$ for $j \geqslant 3$ then the " $\div$ " sign in (15) can be changed to a " $\geqslant$ " sign.

3. The range of values of $t$ for which (15) is likely to be a reasonable approximation is not immediately clear since it depends on the relationships between $R, i(t)$ and $F_{0}$. However, in the special case where $F_{0}$ is a negative exponential distribution we can get a clearer idea of the accuracy of (15). Let us suppose then that $\mathrm{F}_{0}(x)=1-\exp \{-a x\}$ for some $a>0$ so that $m=1 / a$ and $\alpha=2 / a^{2}$. Formula (9) gives

$$
c_{t}=p i(t) /[a-R i(t)] \quad \text { provided } a>R i(t) .
$$

This gives the exact relationship

$$
c_{t}=c_{0} i(t)[1-R / a] /[1-R i(t) / a]
$$

Formula (15) gives the following approximation

$$
c_{t} \doteqdot c_{0} i(t)[1+R i(t) / a] /[1+R / a]
$$

So far in this section we have been concerned with $c_{t}$, the instantaneous rate of premium income, which we have assumed to be continuously variable. We now suppose that in the time interval $[n-1, n]$, where $n$ is a positive integer, a total premium $P_{n}$ is payable at a constant rate throughout the year.

\section{Corollary 2}

Assuming either that $i(t)$ is a non-decreasing function of $t$ or that "ruin" can only occur after an integral number of years, the probability of ultimate ruin for our risk process will be bounded above by $\exp \{-R U\}$ if $P_{n}$ is calculated by the formula

$$
P_{n}=p\left[\int_{n-1}^{n} m_{t}(R) d t-1\right] / R \quad n=1,2, \ldots
$$

This formula for $P_{n}$ gives the following approximation for small values of $n$

$$
P_{n} \doteqdot E\left[Y_{n}\right]+(R / 2) \operatorname{Var}\left[Y_{n}\right]
$$

\section{Proof}

The first part of the corollary is easily proved since (21) can be written

$$
P_{n}=\int_{n-1}^{n} c_{t} d t
$$

where $c_{t}$ is as in (9). Using similar approximations to those used in the proof of 
Corollary 1 we have that

$$
P_{n} \doteqdot p \int_{n-1}^{n}\left[m i(t)+\alpha R i^{2}(t) / 2\right] d t
$$

and so (22) follows from (5) and (6).

\section{Remarks}

1. If $i(t) \geqslant 1$ and $\alpha_{j} \geqslant 0$ for $j \geqslant 3$ the " $\doteqdot$ " sign in (22) can be replaced by a " $\geqslant$ " sign.

2. Formula (21) is equivalent to

$$
P_{n}=(1 / R) \ln \left[E\left[\exp \left\{R Y_{n}\right\}\right]\right]
$$

In other words to keep the probability of ruin below $\exp \{-R U\}$ the annual premium should be calculated using the principle of zero utility with the utility function

$$
u(x)=(1 / R)(1-\exp \{-R x\})
$$

See Gerber (1974).

$$
\text { REFERENCES }
$$

BüHLmanN, H. (1970). Mathematical Methods in Risk Theory, Springer, New York.

GERBER, H. U. (1973). Martingales in Risk Theory, Mitteilungen der Vereinigung Schweizerischer Versicherungsmathematiker, 73, 205-216.

Gerber, H. U. (1974). On Additive Premium Calculation Principles, Astin Bulletin, 7, $215-222$.

Gerber, H. U. (1975). The Surplus Process as a Fair Game-Utilitywise, Astin Bulletin, $8,307-322$.

TAYLOR, G. C. (1979). Probability of Ruin under Inflationary Conditions or under Experience Rating, Astin Bulletin, 10, 149-162. 